\title{
OPN/CD44v6 overexpression in laryngeal dysplasia and correlation with clinical outcome
}

\author{
S Staibano', F Merolla ${ }^{2}$, D Testa ${ }^{3}$, R lovine ${ }^{4}$, M Mascolo', V Guarino², MD Castellone ${ }^{2}$, M Di Benedetto', \\ V Galli', S Motta ${ }^{3}$, RM Melillo', G De Rosa', M Santoro' ${ }^{2}$ and A Celetti*,2 \\ 'Dipartimento di Scienze Biomorfologiche e Funzionali, Università di Napoli 'Federico Il', Naples, Italy; 'Dipartimento di Biologia e Patologia \\ Cellulare e Molecolare, Università di Napoli 'Federico II', Istituto di Endocrinologia ed Oncologia Sperimentale del CNR, Naples, Italy; ${ }^{3}$ Clinica \\ Otorinolaringoiatrica, Seconda Università di Napoli, Naples, Italy; ${ }^{4}$ Dipartimento Assistenziale di Otorinolaringoiatria e Scienze Affini, Università di Napoli \\ 'Federico II', Naples, Italy
}

\begin{abstract}
Laryngeal dysplasia is a common clinical concern. Despite major advancements, a significant number of patients with this condition progress to invasive squamous cell carcinoma. Osteopontin (OPN) is a secreted glycoprotein, whose expression is markedly elevated in several types of cancers. We explored OPN as a candidate biomarker for laryngeal dysplasia. To this aim, we examined OPN expression in 82 cases of dysplasia and in hyperplastic and normal tissue samples. OPN expression was elevated in all severe dysplasia samples, but not hyperplastic samples, with respect to matched normal mucosa. OPN expression levels correlated positively with degree of dysplasia $(P=0.0094)$ and negatively with disease-free survival $(P<0.000 \mathrm{I})$. OPN expression was paralleled by cell surface reactivity for CD44v6, an OPN functional receptor. CD44v6 expression correlated negatively with disease-free survival, as well $(P=0.0007)$. Taken as a whole, our finding identify OPN and CD44v6 as predictive markers of recurrence or aggressiveness in laryngeal intraepithelial neoplasia, and overall, point out an important signalling complex in the evolution of laryngeal dysplasia. British Journal of Cancer (2007) 97, I545- I55 I. doi:I0.I038/sj.bjc.6604070 www.bjcancer.com
\end{abstract}

Published online 6 November 2007

(c) 2007 Cancer Research UK

Keywords: OPN; SCC; dysplasia; marker

Laryngeal squamous cell carcinoma (LSCC) is the most common type of head and neck squamous cell carcinoma (HNSCC). LSCC accounts for $1-2 \%$ of all malignancies diagnosed worldwide (Vokes et al, 1993; Licitra et al, 2003; Mao et al, 2004). Notwithstanding primary prevention, screening, surgical treatment, and radiotherapy, the long-term survival rate of LSCC patients has remained substantially unchanged in the last two decades (Hoffman et al, 1998). Survival of the patients depends on the stage of the disease; therefore, early detection and timely therapy are essential (Vokes et al, 1993; Hoffman et al, 1998; Licitra et al, 2003; Mao et al, 2004).

Laryngeal squamous cell carcinoma usually develops in a multistep process: normal mucosa - dysplasia (laryngeal intraepithelial neoplasia, LIN) - LSCC in situ - invasive LSCC (Rosai et al, 1992; Tabor et al, 2002; Zuckerberg, 2002; Johnson, 2003). Dysplasia is characterised by increased cell growth, cellular atypia (nuclear and nucleolar abnormalities, altered nuclear/cytoplasmatic ratio, and altered cytoplasmatic differentiation), and architectural alteration of the epithelium. Conventionally, the dysplastic changes are graded as mild (LIN I: dysplasia limited to the basal third of the epithelium, few mitoses), moderate (LIN II: dysplasia involving the lower two-thirds of the epithelium, marked nuclear changes, prominent nucleoli, mitoses in the parabasal, and

*Correspondence: Dr A Celetti; E-mail: celetti@unina.it Received I 8 July 2007; revised I October 2007; accepted 9 October 2007; published online 6 November 2007 intermediate layers), and severe (LIN III: dysplasia involving more than two-thirds of the epithelial thickness, nuclear pleomorphism and hyperchromasia, prominent nucleoli, cell crowding, and atypical mitoses). Often, severe dysplasia and in situ carcinoma are grouped in the same category (Rosai et al, 1992; Tabor et al, 2002; Zuckerberg, 2002; Johnson, 2003). Early forms of dysplasia may be reversible if the initial stimuli (like smoke and volatile irritating substances) are removed, while severe dysplasia, if left untreated, is regarded as a precancerous lesion (Rosai et al, 1992; Tabor et al, 2002; Zuckerberg, 2002; Johnson, 2003). For patients with mild or moderate dysplasia, the reported rate of progression to invasive cancer is up to 11.5 and $45 \%$, respectively. In severe dysplasia, higher rates of progression are commonly reported (Rosai et al, 1992; Tabor et al, 2002; Zuckerberg, 2002; Johnson, 2003). The molecular events that induce the evolution of dysplasia to carcinoma are still unknown (Cowan et al, 1992; Zuckerberg, 2002; Perez-Ordonez et al, 2006).

Osteopontin (OPN), also known as SPP1 (secreted phosphoprotein 1), is a highly acidic calcium-binding glycosylated phosphoprotein (Weber, 2001; Rittling and Chambers, 2004; Rangaswami et al, 2006). OPN can function both as cell adhesion molecule and as cytokine. It binds to the cell surface receptors $\alpha \mathrm{v}$ or $\beta 1$-containing integrins and CD44v6 (Weber, 2001; Rittling and Chambers, 2004; Rangaswami et al, 2006), thereby supporting proliferation, chemotaxis, attachment, and migration of many cell types. CD44 is a cell surface glycoprotein that is involved in regulating cell-cell and cell-matrix interactions, migration, and tumour growth and progression (Ponta et al, 2003). CD44 is 
expressed as a standard receptor (CD44s) and in multiple splice isoforms $(\mathrm{CD} 44 \mathrm{v})$, whose expression is altered during tumour growth and progression. Expression of the ' $v 6$ ' variant exon of CD44 is necessary for OPN binding (Ponta et al, 2003). OPN is overexpressed in many human tumours, for example, colon, breast, liver, prostate, gastric, ovarian, lung, thyroid, and kidney carcinomas (Agrawal et al, 2002; Kang et al, 2003; Ye et al, 2003; Schorge et al, 2004; Donati et al, 2005; Guarino et al, 2005; Matusan et al, 2006).

We recently reported that OPN and CD44v6 are overexpressed in full-blown LSCC (Celetti et al, 2005). Here, we have investigated the role of the OPN/CD44v6 axis in laryngeal dysplasia.

\section{MATERIALS AND METHODS}

\section{Study population}

Patients (82 cases: 77 men and 5 women) underwent surgery at the Otolaryngology Department of the University Federico II of Naples between January 1993 and December 2001. The patients' age ranged from 23 to 83 years, with a mean of 62.46 years. Paraffin blocks were retrieved from the files of the Department of Biomorphological and Functional Sciences, Pathology Section, University Federico II of Naples. Each patient agreed to and signed a consent for the treatment of clinical data and tissues for diagnostic and research purposes, according to the guidelines of the Institutional Ethic Committee. For all the patients, clinicopathologic and follow-up data were recorded (Table 1). Before surgery, patients underwent otolaryngological, fibreoptic, and radiological evaluation. The mean follow-up time was 10.1 years (range: 8-13 years). Follow-up consisted in clinical and radiological evaluation at 3-month intervals for the first year and 6-months intervals thereafter. At completion of follow-up, patients were subdivided into alive with absence of relapse (no); alive with evidence of recurrent disease (LIN); and alive with progression of disease to LSCC (SCC).

After surgical resection, tissues were fixed in $10 \%$ neutral buffered formalin and embedded in paraffin blocks. Sections $(4-\mu \mathrm{M}$ thick) were stained with haematoxylin-eosin for histological examination. The pathologic analysis was performed in a blinded fashion to the clinical informations. The cytological evaluation was according to standard criteria (Evans et al, 1986; Gale et al, 2000; Tabor et al, 2003).

Table I Clinicopathological features of studied laryngeal intraepithelial neoplasia (LIN) patients

\begin{tabular}{lc}
\hline Characteristics & Total (\%) \\
\hline No. of subjects & \\
Male & $77(94)$ \\
Female & $5(6)$ \\
Disease site & \\
$\quad$ Glottis-hypoglottis & $43(53)$ \\
Supraglottis & $39(47)$ \\
Degree of dysplasia & \\
Mild & $21(47)$ \\
Moderate & $8(19)$ \\
Severe & $53(64)$ \\
Relapse & \\
No & $35(43)$ \\
LIN & $10(12)$ \\
SCC & $37(45)$ \\
\hline SCC squ
\end{tabular}

$\mathrm{SCC}=$ squamous cell carcinoma.

\section{Immunohistochemistry}

Four-micromolar thick serial sections, mounted on poly-L-lysinecoated glass slides, were dewaxed, rehydrated through multiple graded ethanol solutions, treated with $3 \%$ hydrogen peroxide for $5 \mathrm{~min}$ to inactivate endogenous peroxidases, and washed in distilled water. After antigen retrieval (microwave oven $5 \mathrm{~min} \times 3$ times, in $1 \%$ citrate buffer), nonspecific binding was blocked by incubation ( $2 \mathrm{~h}$ at room temperature) with $1.5 \%$ blocking serum. Slides were first incubated with anti-OPN (final concentration: $5 \mu \mathrm{g} \mathrm{ml}^{-1}$ ) (10A16; Assay Designs, Ann Arbor, MI, USA) or antiCD44v6 (dilution of $1: 100$ ) (NCL-CD44v6, clone VFF-7; Novocastra Laboratories Ltd, Newcastle upon Tyne, UK) monoclonal antibodies and then with biotinylated anti-IgG and the premixed avidin-biotin complex (overnight at $4^{\circ} \mathrm{C}$ ) (Vectastain $\mathrm{ABC}$ kits; Vector Laboratories, Burlingame, CA, USA). The immune reaction was revealed with $0.06 \mathrm{mmoll}^{-1}$ diaminobenzidine (DAB-DAKO, Carpinteria, CA, USA) and $2 \mathrm{mmol}^{-1}$ hydrogen peroxide. Finally, slides were counterstained with haematoxylin and coverslipped with a synthetic mounting media. Control slides in the presence of preimmune serum were included for each staining as an additional negative control. Anti-OPN antibody was preincubated with a fivefold molar excess of OPN peptide to ascertain specificity of the reaction.

The results of the immunohistochemical staining were evaluated separately and in a blinded fashion by two pathologists. Five representative microscopic areas at $\times 400$ magnification were randomly selected for examination. Expression of OPN was semiquantitatively assessed as percentage of positive cells with respect to the total number of epithelial cells. The samples were assigned to one of the four following categories: 0 (absence of positive cells $) ;+(<10 \%$ of positive cells $) ;++(10-50 \%$ of positive cells); and $+++(>50 \%$ of positive cells). Staining of CD44v6 was classified as 'lower' (lower third) (L), 'lower and middle' (up to two-thirds of the thickness of the epithelium) (M), and 'full thickness' (F).

\section{Statistical analysis}

The Pearson's $\chi^{2}$ test was used to assess the statistical significance of the frequency distribution of all categories of OPN or CD44v6 expression by degree of dysplasia or by relapse. Differences were significant with $P$-value $<0.05$.

Nonparametric Spearman's correlation coefficient method was used to assess the statistical significance of the correlation between OPN expression vs CD44v6 positivity. A test was run for all the patients' cohort combined or grouped by degree of dysplasia or by type of relapse. Correlations were significant when $P$-value was $<0.05$. Disease-free survival curves of the patients were calculated using the Kaplan-Meier method, and analysis was performed by the log-rank test. Differences were significant when $P<0.05$. In this analysis, a group of 31 patients has been censored for lack of data. Statistical analysis was performed using the JMP software program (version 5.1.1; SAS Institute Inc., Austin, TX, USA).

\section{RESULTS}

\section{Immunohistochemical detection of OPN and CD44v6 in laryngeal dysplasia}

Eighty-two laryngeal samples with different degree of dysplasia (Table 1) and the matched normal mucosa were tested for OPN expression by immunohistochemistry with an anti-OPN-specific monoclonal antibody. Representative stainings are shown in Figure 1, and the entire data set is reported in Table 2. OPN was virtually undetectable $(<2.0 \%$ of the cells) in normal tissue $(n=10)$. Dysplastic areas showed different degrees of OPN positivity. In most (76\%) of mild dysplasia cases, only few cells 

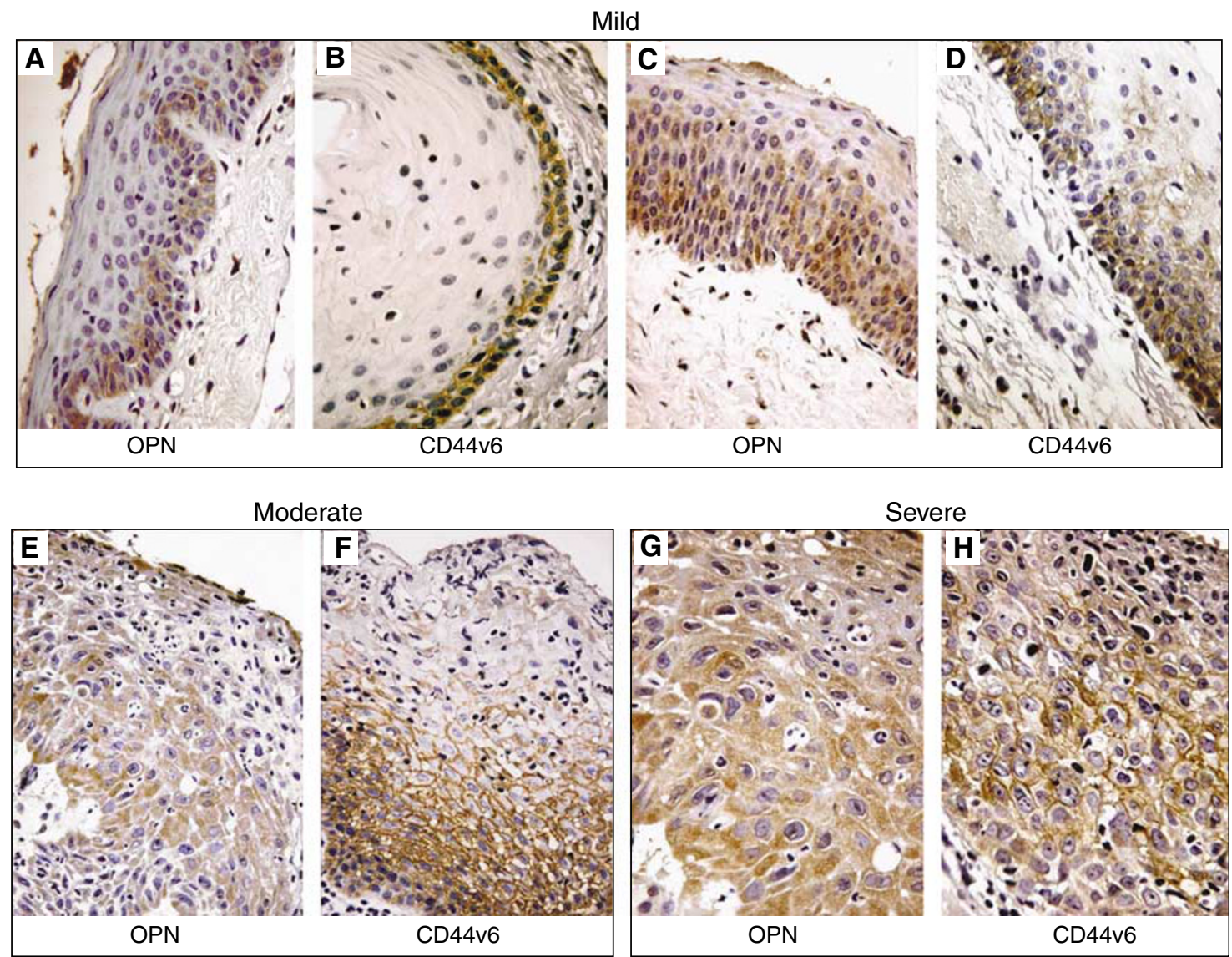

Figure I (A and B) A representative case of mild degree dysplasia showing weak immunostaining for OPN $(\mathbf{A})$ and CD44v6 positivity restricted to the lower third of the epithelium $(\mathbf{B})(\times 150)$. (C and D) Another mild dysplasia sample showing stronger immunostaining for both the markers $(\times \mid 50)$. $(\mathbf{E}$ and $\mathbf{F})$ A representative case of moderate dysplasia showing a strong immunostaining for OPN (E) and a CD44v6 positivity involving the lower twothirds of the epithelium $(\mathbf{F})(\times 250)$. (G and $\mathbf{H})$ A case of high-grade dysplasia showing strong immunostaining for OPN (G) and CD44v6 positivity up to the upper third of the epithelium $(\mathbf{H})(\times 400)$.

Table 2 OPN and CD44v6 positivity in laryngeal intraepithelial neoplasia at different degree

\begin{tabular}{|c|c|c|}
\hline LIN & OPN positivity ${ }^{a}$ & CD44v6 positivity \\
\hline Mild (2I) & $\begin{array}{ll}16 / 21 & (+) \\
1 / 21 & (++) \\
4 / 2 \mid & (+++)\end{array}$ & $\begin{array}{c}|6 / 2|(\mathrm{L}) \\
2 / 21(\mathrm{M}) \\
3 / 21(\mathrm{~F})\end{array}$ \\
\hline Moderate (8) & $\begin{array}{l}3 / 8(+) \\
1 / 8(++) \\
4 / 8(+++)\end{array}$ & $\begin{array}{l}2 / 8(\mathrm{~L}) \\
3 / 8(\mathrm{M}) \\
3 / 8(\mathrm{~F})\end{array}$ \\
\hline Severe (53) & $\begin{array}{l}16 / 53(+) \\
11 / 53(++) \\
26 / 53(+++)\end{array}$ & $\begin{array}{l}15 / 53(\mathrm{~L}) \\
12 / 53(\mathrm{M}) \\
26 / 53(\mathrm{~F})\end{array}$ \\
\hline
\end{tabular}

OPN = osteopontin. ${ }^{a}$ Osteopontin and CD44v6 expression were assessed by immunohistochemistry and scored respectively as follows: $+=<10 \%$ positive cells; $++=10-50 \%$ positive cells; and $+++=50-100 \%$ positive cells; $L=$ lower; $M=$ lower and middle; and $F=$ full-thickness involvement of the epithelial layers,

$(+)$ were positive (Figure $1 \mathrm{~A}$ ), while $50 \%$ of moderate and severe dysplasia samples had intense $(+++$ ) OPN staining (Figures $1 \mathrm{E}$ and G). Only $20 \%$ cases of mild dysplasia were highly positive for OPN $(+++)$; interestingly, in these samples, OPN expression coexisted with a diffuse CD44v6 staining (see below) (Figures 1C and D).

The samples were also analysed for the expression of CD44v6, the receptor that is involved in OPN binding (Table 2). Only basal cells ( $\mathrm{L}$ category) were CD44v6 positive in $76 \%$ of mild dysplasia samples (Figure 1B). Instead, in moderate dysplasia, CD44v6 positivity was found in the basal two-thirds ( $M$ category) (Figure 1F) or even full thickness (F category) of the epithelium. Finally, $50 \%$ of severe dysplasia samples showed a full-thickness (F category) CD44v6 staining (Figure 1H). The association between OPN and CD44v6 immunoreactivity resulted significant when analysed by the Spearman's rank correlation test (Table 3A). The frequency distribution of OPN positivity or CD44v6 immunoreactivity by degree of dysplasia resulted highly significant at Pearson's $\chi^{2}$ test (Table 4A).

Foci of squamous metaplasia of laryngeal cylindric-cell-lined areas were almost constantly present in our samples. Metaplastic areas $(n=20)$ were almost constantly negative for OPN and CD44v6 staining (Figures 2A and B). Only in few (5\%) cases, we observed an intense $(+++)$ OPN staining paralleled by fullthickness CD44v6 positivity in the squamous metaplastic cells (Figures 2C and D). Although the ultimate statistical relevance of this finding is still to be verified on larger series of cases, it is interesting to note that these OPN- and CD44v6-positive metaplasia areas were found in patients with a history of development of SCC at the follow-up.

\section{OPN and CD44v6 expression levels in laryngeal dysplasia negatively correlate with disease-free survival}

The disease-free survival rate in patients affected by laryngeal dysplasia negatively correlated with intense OPN staining and fullthickness CD44v6 positivity. As shown by the Kaplan-Meier 
survival curves reported in Figure 3A, the 8-years disease-free survival was 94 and $91 \%$ for OPN $(+)$ - and OPN $(++)$-positive cases, respectively, and $33 \%$ for OPN $(+++)$-positive cases (two-sided log-rank test, $P<0.0001$; Figure 4A). Relative to CD44v6 expression, the probability of recurrence was 94 and $75 \%$ for cases that showed basal (L), or basal and middle (M) staining, respectively, and $38 \%$ for patient that had full-thickness (F) positivity (two-sided log-rank test, $P=0.007$; Figure $4 \mathrm{~B}$ ).

Table 3A Correlation of osteopontin and CD44v6 expression in all laryngeal intraepithelial neoplasia (LIN) patients combined or grouped by degree of dysplasia

\begin{tabular}{lcc}
\hline LIN & $\boldsymbol{r}_{\mathbf{s}}$ & $\boldsymbol{P}_{\mathbf{s}}$ \\
\hline Combined (82) & 0.8231 & $<0.000 \mathrm{I}$ \\
Mild (2I) & 0.9941 & $<0.0001$ \\
Moderate (8) & 0.2622 & $=0.5304$ \\
Severe (53) & 0.7957 & $<0.000$ I \\
\hline
\end{tabular}

Note: Correlation between osteopontin and CD44v6 expression in LIN patients analysed by Spearman's rank correlation test. Correlation coefficient $\left(r_{s}\right)$ and $P_{s}$ are shown $\left(P_{\mathrm{s}}<0.05\right.$ was considered significant).

Table 3B Correlation of osteopontin and CD44v6 expression in all laryngeal intraepithelial neoplasia (LIN) patients combined or grouped by relapse

\begin{tabular}{lcc}
\hline Relapse & $\boldsymbol{r}_{\mathbf{s}}$ & $\boldsymbol{P}_{\mathbf{s}}$ \\
\hline Combined (82) & 0.8231 & $<0.0001$ \\
no (35) & 0.8133 & $<0.0001$ \\
LIN (10) & 0.5976 & $=0.0734$ \\
SCC (37) & -0.7892 & $<0.0001$ \\
\hline
\end{tabular}

SCC = squamous cell carcinoma. Note: Absence of relapse (no), recurrence of dysplasia (LIN), progression to carcinoma (SCC). Correlation between osteopontin and CD44v6 expression in LIN patients analysed by Spearman's rank correlation test. Correlation coefficient $\left(r_{\mathrm{s}}\right)$ and $P_{\mathrm{s}}$ are shown $\left(P_{\mathrm{s}}<0.05\right.$ was considered significant).
At the Pearson's test, the frequency distribution of OPN and CD44v6 expression levels were significantly correlated with relapse (Table 4B).

Moreover, the correlation between OPN and CD44v6 expression in patients with absence of relapse, with recurrence of dysplasia, or with progression to LSCC resulted very significant at the Spearman's rank correlation test (Table 3B). Finally, the contingency analysis showed that the frequency distribution of OPN by CD44v6 were highly significant in combined or grouped types of relapse (Table 4C).

\section{DISCUSSION}

An in-depth understanding of the factors involved in the initial steps of LSCC development will facilitate the prevention and diagnosis of this condition. Currently, histological grading and the

Table 4 Pearson's test

\begin{tabular}{|c|c|c|}
\hline & $\chi^{2}$ & $P$ \\
\hline \multicolumn{3}{|c|}{$\begin{array}{l}\text { A. Contingency analysis of osteopontin (OPN) and CD } 44 v 6 \text { positivity by degree of } \\
\text { dysplasia }\end{array}$} \\
\hline OPN & 13.425 & 0.0094 \\
\hline CD44v6 & 16.198 & 0.0028 \\
\hline \multicolumn{3}{|c|}{ B. Contingency analysis of OPN and CD44v6 positivity by relapse (no, LIN, SCC) } \\
\hline OPN & 21.780 & $<0.0002$ \\
\hline CD44v6 & 9.567 & 0.0484 \\
\hline \multicolumn{3}{|c|}{$\begin{array}{l}\text { C. Contingency analysis of OPN positivity by CD } 44 v 6 \text { expression in all laryngeal } \\
\text { intraepithelial neoplasia combined or grouped by relapse }\end{array}$} \\
\hline Combined (82) & 73.026 & $<0.0001$ \\
\hline No (35) & 32.694 & $<0.0001$ \\
\hline LIN (10) & 10.000 & 0.0067 \\
\hline $\operatorname{sCC}(37)$ & 34.857 & $<0.0001$ \\
\hline
\end{tabular}

LIN = laryngeal intraepithelial neoplasia; SCC = squamous cell carcinoma. Note: Absence of relapse (no), recurrence of dysplasia (LIN), and progression to carcinoma $(\mathrm{SCC})$
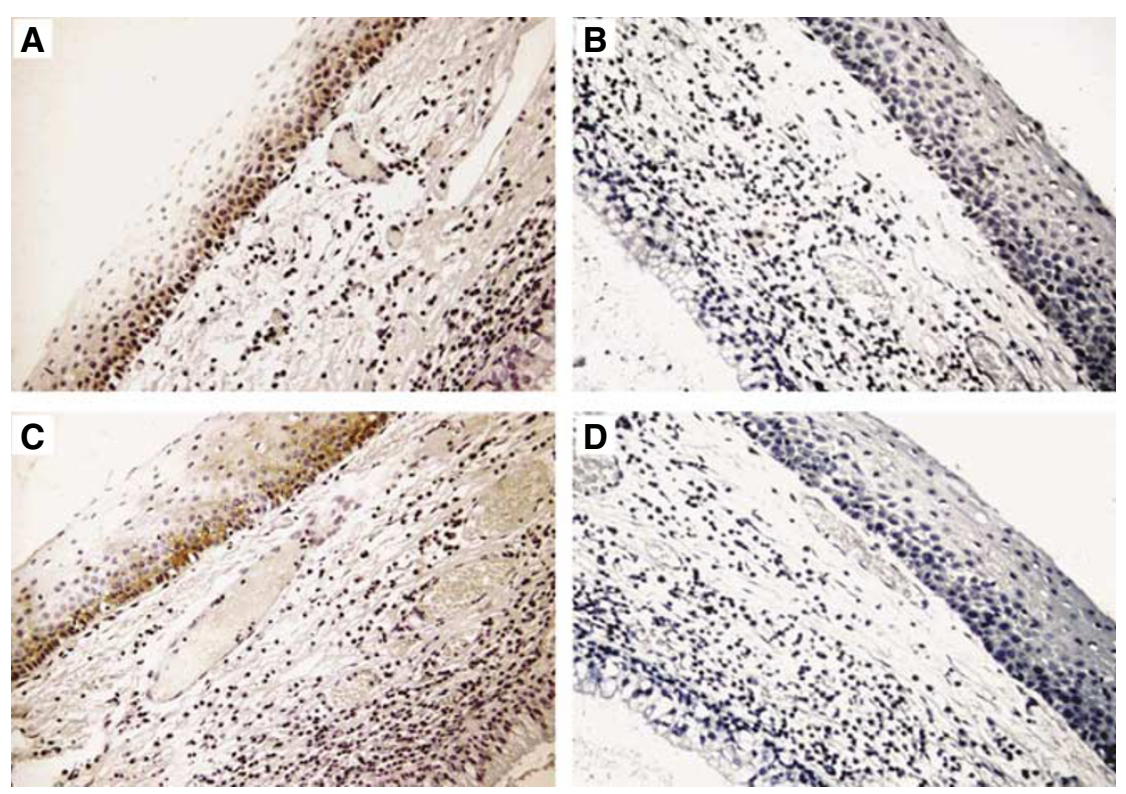

Figure 2 (A and $\mathbf{C})$ A representative case of mild degree dysplasia showing weak immunostaining for OPN $(\mathbf{A})$ and CD44v6 positivity restricted to the lower third of the epithelium $(\mathbf{C})(\times 150)$. (B and $\mathbf{D})$ The same samples as in $(\mathbf{A})$ negative for OPN immunostaining after incubation with isotype control antiserum $(\mathbf{B})(\times$ I50), and the same sample as in $(\mathbf{C})$ negative for CD44v6 immunostaining after incubation with isotype control antiserum $(\mathbf{D})(\times$ I50), 


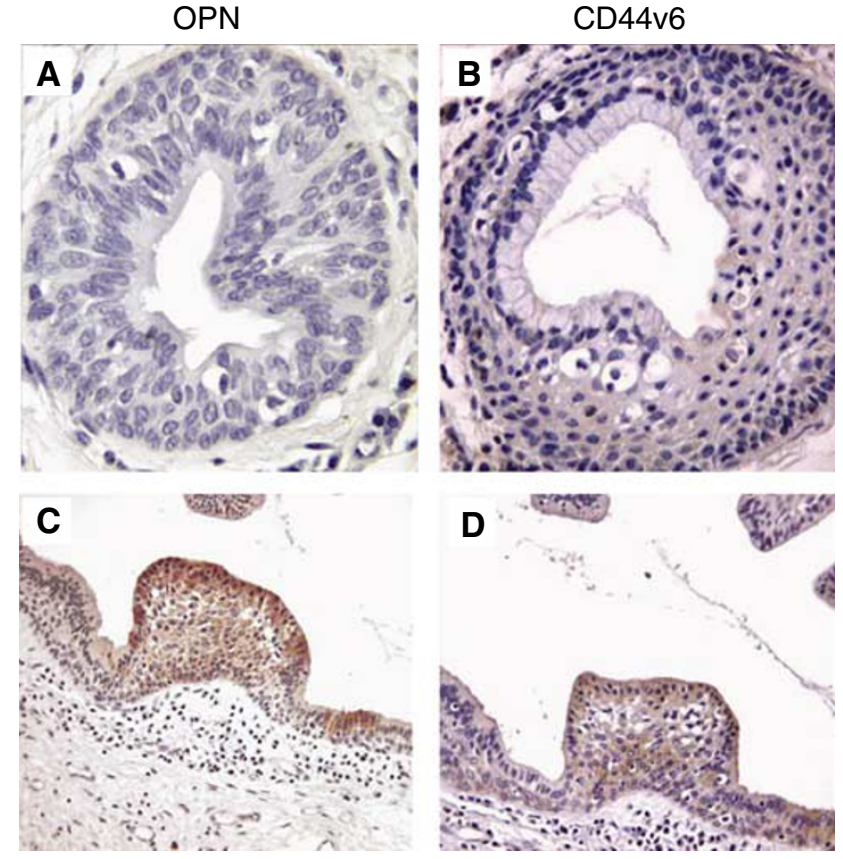

Figure 3 (A and $\mathbf{B})$ An area of incomplete squamous metaplasia, negative for OPN (A), and CD44v6 (B) immunostaining ( $\times 250)$. (C and D) Another case of incomplete squamous metaplasia (patient with a history of laryngeal SCC), showing an intense OPN (C) and CD44v6 (D) immunostaining $(\times 106)$.

topographical extension of laryngeal dysplasia are used to predict the risk for cancer and to determine the treatment strategy (Rosai et al, 1992; Zuckerberg, 2002; Johnson, 2003). Although significant efforts have been made to identify molecular markers of the clinical outcome of premalignant laryngeal lesion, still neither single nor combination of markers is accepted in clinical practice (Tabor et al, 2002).

We have previously reported that both OPN and CD44v6 are highly expressed in invasive LSCC (Celetti et al, 2005). Here, we have explored whether the same ligand/receptor pair is involved in the premalignant phases as well. To this aim, we have investigated OPN and CD44v6 in a set of laryngeal dysplasia samples and correlated their expression level to histological grading and clinical outcome. Both OPN and CD44v6 were consistently overexpressed in dysplastic but not hyperplastic or metaplastic mucosa. Dysplasia, in particular high-grade dysplasia, is regarded as a preneoplastic condition (Rosai et al, 1992). Importantly, OPN/ CD44v6 overexpression was significantly correlated with the degree of dysplasia, type of recurrence, and reduced disease-free survival. Taken together, these findings suggest that OPN signalling through CD44v6 may play a role in the establishment of dyplastic changes in the laryngeal epithelium. Interestingly, it has been recently reported that genetic deletion of OPN in transgenic mice did not change the rate of hyperplasia formation but caused a reduction of benign papilloma formation after the two-stage skin chemical carcinogenesis protocol; thus, also in this experimental model system, OPN is involved in the early phases of tumorigenesis (Hsieh et al, 2006).

Osteopontin is able to engage several cell surface receptors, including integrins and CD44 variants. In particular, OPN binds CD44 proteins that contain v6-encoded sequences, and OPN/ CD44v6 binding has been implicated in carcinogenesis (Ponta et al, 2003). Here, we show that OPN expression levels were paralleled by intense expression of CD44v6; at contingency analysis, the frequency distribution of OPN expression by CD44v6 positivity resulted highly significant at Pearson's test;
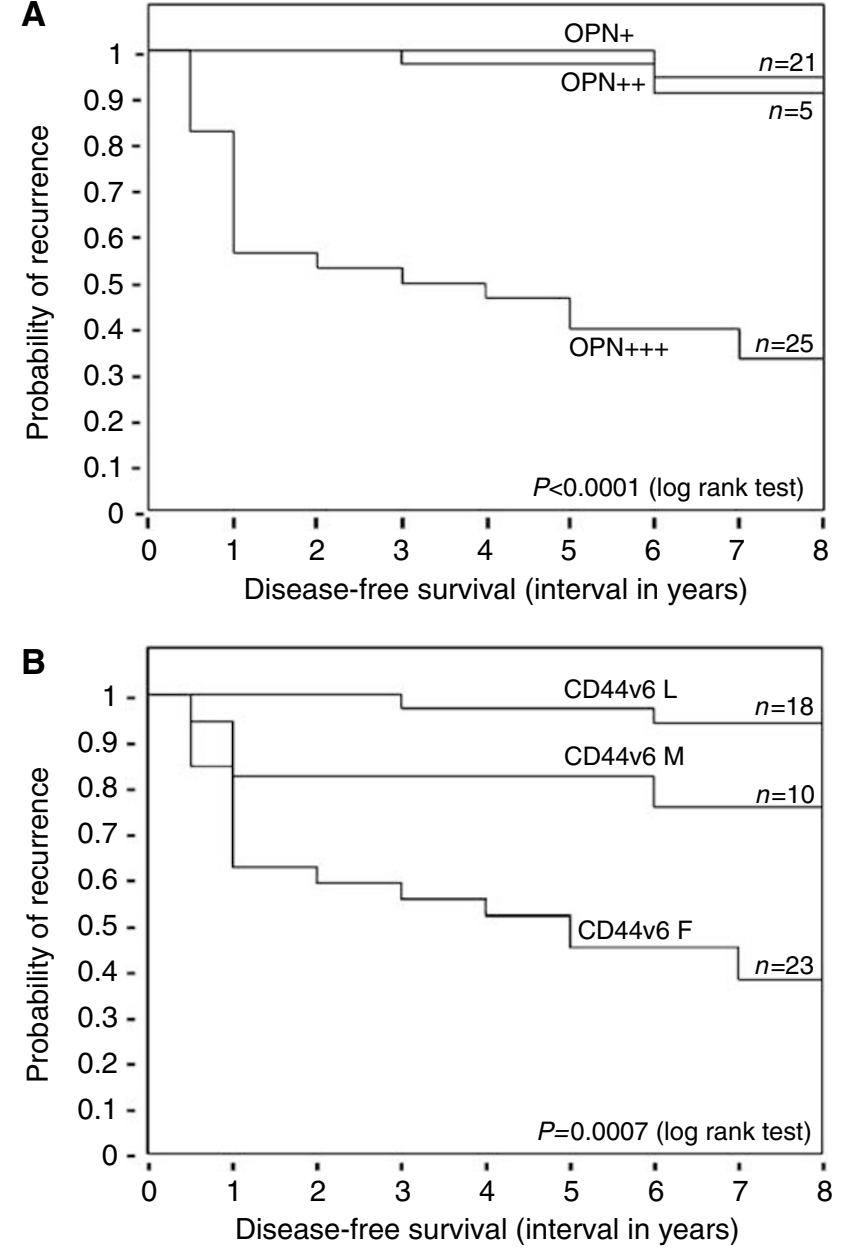

Figure 4 Osteopontin and CD44v6 staining in laryngeal dysplasia negatively correlates with disease-free survival. Kaplan-Meier survival plots for patients grouped on the basis of the OPN (A) or CD44v6 (B) expression level. (A) Patients were stratified into three categories $(+\quad(n=2 \mathrm{I}),++(n=5)$, and $+++(n=25))$ based on OPN immunostaining intensity. (B) Patients were stratified into three categories $(L(n=18), M(n=10)$, and $F(n=23)$ based on the thickness of CD44v6 staining). The $P$-value was determined by a two-sided log-rank test.

moreover, the association between OPN and CD44v6 immunoreactivity was highly significant at the Spearman's correlation coefficient test, suggesting that CD44v6 is at least one of the functional OPN receptors in laryngeal dysplasia.

To investigate whether the OPN/CD44v6 overexpression was causally related with dysplasia, cytological changes induced by OPN stimulation of primary human keratinocytes, obstructed by CD44 blockade, have been observed (Celetti A et al, in preparation). Addressing CD44 as a functional receptor for OPN would be important to explore the molecular mechanism underlying dysplastic changes induced by the OPN/CD44 axis. It is known that CD44 triggering stimulates diverse signalling pathways, including activation of ERK (Bourguignon et al, 2005), RAC (Teramoto et al, 2005), and RHO (Bourguignon et al, 2003), as well as secretion of soluble factors, like cytokines and metalloproteinases (Zhang et al, 2002; Bourguignon et al, 2003; Murphy et al, 2005). These pathways are potentially involved in dysplastic changes induced by OPN/CD44v6.

A model for the initiation and progression of colorectal cancer has become a paradigm for other human solid tumours (Fearon and Vogelstein, 1990). Like colorectal cancer, HNSCC is thought to 
progress through a series of well-defined clinical and histopathological stages. While not all of the specific mutations required for progression have been delineated, a working molecular model has been proposed (Silverman, 2003). The loss of chromosomal regions $3 \mathrm{p}$ and $9 \mathrm{p} 21$ are among the first identified genetic changes (Mao et al, 1996; Sanz-Ortega et al, 2003). In particular, lossof-heterozygosity $(\mathrm{LOH})$ at $9 \mathrm{p} 21$ in conjunction with promoter hypermethylation results is the inactivation of the CDKN2A gene, coding for the cyclin-dependent kinase inhibitor 2A (p16INK4). This alteration occurs prior to the development of histologic atypia and is associated with the transition from normal to hyperplastic/ metaplastic mucosa (Papadimitrakopoulou et al, 2001; SanzOrtega et al, 2003). Subsequent LOH at 17p with mutation of the TP53 tumour suppressor gene is associated with progression to dysplasia (Boyle et al, 1993). The overexpression of the EGF receptor is also an early event in carcinogenesis (Rubin Grandis et al, 1998). Amplification and overexpression of the CCND1 gene, encoding cyclin D1 is a common late event in HNSCC formation
(Michalides et al, 1995; Izzo et al, 1998; Chatrath et al, 2006). Our findings suggest that the upregulation of the OPN/CD44v6 axis is an additional early event during the progression of laryngeal dysplasia. Thus, early immunocytochemical detection of OPN and CD44v6 can be exploited to set a screening test for laryngeal dysplasia. Moreover, perturbation of OPN/CD44v6 signalling may represent a promising novel strategy to prevent progression of laryngeal preneoplastic lesions.

\section{ACKNOWLEDGEMENTS}

We thank Giancarlo Vecchio for continuous support. We are grateful to JA Gilder for text editing. This study was supported by the Associazione Italiana per la Ricerca sul Cancro (AIRC), the NOGEC (Naples OncoGEnomic Center), and the Italian Ministero della Salute.

\section{REFERENCES}

Agrawal D, Chen T, Irby R, Quackenbush J, Chambers AF, Szabo M, Cantor A, Coppola D, Yeatman TJ (2002) Osteopontin identified as lead marker of colon cancer progression, using pooled sample expression profiling. J Natl Cancer Inst 94: 513-521

Bourguignon LY, Gilad E, Rothman K, Peyollier K (2005) HyaluronanCD44 interaction with IQGAP1 promotes Cdc42 and ERK signaling leading to actin binding, Elk-1/estrogen receptor transcriptional activation and ovarian cancer progression. J Biol Chem 280: 11961-11972

Bourguignon LY, Singleton PA, Zhu H, Diedrich F (2003) Hyaluronanmediated CD44 interaction with RhoGEF and Rho kinase promotes Grb2-associated binder-1 phosphorylation and phosphatidylinositol 3-kinase signaling leading to cytokine (macrophage-colony stimulating factor) production and breast tumor progression. J Biol Chem 278: $29420-29434$

Boyle JO, Hakim J, Koch W, van der Riet P, Hruban RH, Roa RA, Correo R, Eby YJ, Ruppert JM, Sidransky D (1993) The incidence of p53 mutations increases with progression of head and neck cancer. Cancer Res 53: $4477-4480$

Celetti A, Testa D, Staibano S, Merolla F, Guarino V, Castellone MD, Iovine R, Mansueto G, Somma P, De Rosa G, Galli V, Melillo RM, Santoro M (2005) Overexpression of the cytokine osteopontin identifies aggressive laryngeal squamous cell carcinomas and enhances carcinoma cell proliferation and invasiveness. Clin Cancer Res 11: 8019-8027

Chatrath P, Scott IS, Morris LS, Davies RJ, Bird K, Vowler SL, Coleman N (2006) Immunohistochemical estimation of cell cycle phase in laryngeal neoplasia. Br J Cancer 95: 314-321

Cowan JM, Beckett MA, Ahmed-Swan S, Weichselbaum RR (1992) Cytogenetic evidence of the multistep origin of head and neck squamous cell carcinomas. J Natl Cancer Inst 84: 793-797

Donati V, Boldrini L, Dell'Omodarme M, Prati MC, Faviana P, Camacci T, Lucchi M, Mussi A, Santoro M, Basolo F, Fontanini G (2005) Osteopontin expression and prognostic significance in non-small cell lung cancer. Clin Cancer Res 11: 6459-6465

Evans DMD, Hudson EA, Brown CL, Boddington MM, Hughes HE, Mackenzie EF, Marshall T (1986) Terminology in gynecological cytopathology: report of the working party of the British Society for Clinical Cytology. J Clin Pathol 39: 933-944

Fearon ER, Vogelstein B (1990) A genetic model for colorectal tumorigenesis. Cell 61: $759-767$

Gale N, Kambic V, Michaels L, Cardesa A, Hellquist H, Zidar N, Poljak M (2000) The Ljubljana classification: a practical strategy for the diagnosis of laryngeal precancerous lesions. Adv Anat Pathol 7: 240-251

Guarino V, Faviana P, Salvatore G, Castellone MD, Cirafici AM, De Falco V, Celetti A, Giannini R, Basolo F, Melillo RM, Santoro M (2005) Osteopontin is overexpressed in human papillary thyroid carcinomas and enhances thyroid carcinoma cell invasiveness. J Clin Endocrinol Metab 90: $5270-5278$

Hoffman HT, Karnell LH, Funk GF, Robinson RA, Menck HR (1998) The National Cancer Data Base report on cancer of the head and neck. Arch Otolaryngol Head Neck Surg 124: $951-962$
Hsieh YH, Juliana MM, Hicks PH, Feng G, Elmets C, Liaw L, Chang PL (2006) Papilloma development is delayed in osteopontin-null mice: implicating an antiapoptosis role for osteopontin. Cancer Res 66: 71197127

Izzo JG, Papadimitrakopoulou VA, Li XQ, Ibarguen H, Lee JS, Ro JY, El-Naggar A, Hong WK, Hittelman WN (1998) Dysregulated cyclin D1 expression early in head and neck tumorigenesis: in vivo evidence for an association with subsequent gene amplification. Oncogene 17: $2313-2322$

Johnson FL (2003) Management of advanced premalignant laryngeal lesions. Curr Opin Otolaryngol Head Neck Surg 11: $462-466$

Kang Y, Siegel PM, Shu W, Drobnjak M, Kakonen SM, Cordón-Cardo C, Guise TA, Massagué J (2003) A multigenic program mediating breast cancer metastasis to bone. Cancer Cell 3: 537-549

Licitra L, Bernier J, Grandi C, Locati L, Merlano M, Gatta G, Lefebvre JL (2003) Cancer of the larynx. Crit Rev Oncol Hematol 147: 65-80

Mao L, Hong WK, Papadimitrakopoulou VA (2004) Focus on head and neck cancer. Cancer Cell 5: $311-316$

Mao L, Lee JS, Fan YH (1996) Frequent microsatellite alterations at chromosomes 9p21 and 3p14 in oral premalignant lesions and their value in cancer risk assessment. Nat Med 6: 682-685

Matusan K, Dordevic G, Stipic D, Mozetic V, Lucin K (2006) Osteopontin expression correlates with prognostic variables and survival in clear cell renal cell carcinoma. J Surg Oncol 94: 325-331

Michalides R, van Veelen N, Hart A, Loftus B, Wientjens E, Balm A (1995) Overexpression of cyclin D1 correlates with recurrence in a group of forty-seven operable squamous cell carcinomas of the head and neck. Cancer Res 55: 975 -978

Murphy JF, Lennon F, Steele C, Kelleher D, Fitzgerald D, Long AC (2005) Engagement of CD44 modulates cyclooxygenase induction, VEGF generation, and cell proliferation in human vascular endothelial cells. FASEB J 19: $446-448$

Papadimitrakopoulou VA, Izzo J, Mao L, Keck J, Hamilton D, Shin DM, ElNaggar A, den Hollander P, Liu D, Hittelman WN, Hong WK (2001) Cyclin D1 and p16 alterations in advanced premalignant lesions of the upper aerodigestive tract: role in response to chemoprevention and cancer development. Clin Cancer Res 7: 3127-3134

Perez-Ordonez B, Beauchemin M, Jordan RC (2006) Molecular biology of squamous cell carcinoma of the head and neck. J Clin Pathol 59: 445-453

Ponta H, Sherman L, Herrlich PA (2003) CD44: from adhesion molecules to signalling regulators. Nat Rev Mol Cell Biol 4: 33-45

Rangaswami H, Bulbule A, Kundu GC (2006) Osteopontin: role in cell signaling and cancer progression. Trends Cell Biol 16: 79-87

Rittling SR, Chambers AF (2004) Role of osteopontin in tumour progression. Br J Cancer 90: 1877 - 1881

Rosai J, Carcangiu ML, DeLellis RA (1992) Atlas of Tumor Pathology Tumors of the Larynx, 3rd series. Washington: Armed Forces Institute of Pathology

Rubin Grandis J, Melhem MF, Gooding WE, Day R, Holst VA, Wagener MM, Drenning SD, Tweardy DJ (1998) Levels of TGF-alpha and EGFR 
protein in head and neck squamous cell carcinoma and patient survival. J Natl Cancer Inst 90: 824-832

Sanz-Ortega J, Valor C, Saez MC, Ortega L, Sierra E, Poch J, Hernández S, Sanz-Esponera J (2003) 3p21, 5q21, 9p21 and 17p13 allelic deletions accumulate in the dysplastic spectrum of laryngeal carcinogenesis and precede malignant transformation. Histol Histopathol 18: 1053-1057

Schorge JO, Drake RD, Lee H, Skates SJ, Rajanbabu R, Miller DS, Kim JH, Cramer DW, Berkowitz RS, Mok SC (2004) Osteopontin as an adjunct to CA125 in detecting recurrent ovarian cancer. Clin Cancer Res 10: $3474-3478$

Silverman S (2003) Oral Cancer Hamilton. Ontario: BD Dekker

Tabor MP, Braakhuis BJ, van der Wal JE, van Diest PJ, Leemans CR, Brakenhoff RH, Kummer JA (2003) Comparative molecular and histological grading of epithelial dysplasia of the oral cavity and the oropharynx. J Pathol 199: 354-360

Tabor MP, Brakenhoff RH, Ruijter-Schippers HJ, Van Der Wal JE, Snow GB, Leemans CR, Braakhuis BJ (2002) Multiple head and neck tumours frequently originate from a single preneoplastic lesion. Am J Pathol 161: $1051-1060$
Teramoto H, Castellone MD, Malek RL, Letwin N, Frank B, Gutkind JS, Lee NH (2005) Autocrine activation of an osteopontin-CD44-Rac pathway enhances invasion and transformation by H-RasV12. Oncogene 24: 489-501

Vokes EE, Weichselbaum RR, Lippman SM, Hong WK (1993) Head and neck cancer. $N$ Engl J Med 328: $184-194$

Weber GF (2001) The metastasis gene osteopontin: a candidate target for cancer therapy. Biochim Biophys Acta 1552: $61-85$

Ye QH, Qin LX, Forgues M, He P, Kim JW, Peng AC, Simon R, Li Y, Robles AI, Chen Y, Ma ZC, Wu ZQ, Ye SL, Liu YK, Tang ZY, Wang XW (2003) Predicting hepatitis B virus-positive metastatic hepatocellular carcinomas using gene expression profiling and supervised machine learning. Nat Med 9: 416-423

Zhang Y, Thant AA, Machida K, Ichigotani Y, Naito Y, Hiraiwa Y, Senga T, Sohara Y, Matsuda S, Hamaguchi M (2002) Hyaluronan-CD44s signaling regulates matrix metalloproteinase-2 secretion in a human lung carcinoma cell line QG90. Cancer Res 62: 3962-3965

Zuckerberg L (2002) The molecular basis of displasia. Semin Diagn Pathol 19: $48-53$ 